\title{
Retrospective Analysis of the Risk Factors of Peri-implantitis
}

\author{
Nathan Anderson ${ }^{1}$, Adam Lords ${ }^{2}$, Ronald Laux ${ }^{3}$, Wendy Woodall ${ }^{4}$, Neamat Hassan Abubakr ${ }^{5}$
}

\begin{abstract}
Aim and objective: Peri-implantitis is a key concern for dental implants and the main common reason for implant failure. This investigation evaluated the risk factors and their implications on peri-implantitis.

Materials and methods: A retrospective search of the patients' clinical notes was performed to identify the documented cases of peri-implantitis. The inclusion criteria encompassed patients who were 18 years and older and were seen at the School of Dental Medicine, University of Nevada, Las Vegas, from January 2014 through September 2018. The search revealed that the number of peri-implantitis cases was 28 , with an overall 45 implants. Data were collected and analyzed using the Chi-square test.

Results: Total 28 patients presented with peri-implantitis. The distribution of males to females with peri-implantitis was 60.7 and $39.3 \%$, respectively. The highest number of patients $(21.4 \%)$ presenting with peri-implantitis fell within the age range of $65-69$ years; $53.3 \%$ of periimplantitis cases were in the maxillary arch. The predilection area for peri-implantitis was the mandibular first molar (24.4\%). Periodontitis was the most significant cause $(60.7 \%)$; respiratory diseases $(42.9 \%)$ followed by hypertension $(28.6 \%)$ were the most prevalent medical conditions in the studied population. Peri-implantitis occurred most frequently among Caucasians (62.7\%), followed by Hispanics (29\%).

Conclusion: Within the limitations of the current evaluation, findings support previous claims that periodontitis remains the strongest predictor of peri-implantitis. A correlation may exist between peri-implantitis and the location of the implant and respiratory disease.

Clinical significance: The implant location, the presence of periodontitis, and respiratory diseases are considered to be risk factors for peri-implantitis.

Keywords: Dental implant, Peri-implantitis, Periodontitis, Respiratory diseases.

The Journal of Contemporary Dental Practice (2020): 10.5005/jp-journals-10024-2973
\end{abstract}

\section{INTRODUCTION}

Implant therapy is an increasingly popular treatment utilized by dental professionals with success rates frequently reported above $90 \%{ }^{1,2}$ However, certain conditions have been identified as contributing to an increased risk of complications and implant failure, the most predominant being peri-implantitis. ${ }^{3,4}$ Periimplantitis is generally defined as the inflammatory process distinguished by inflammation of the mucosa adjacent to the dental implants and by loss of supporting bone. ${ }^{5-7}$ The frequent diagnosis of peri-implant mucositis is clinical inflammation signs, such as bleeding on suppuration or probing. Peri-implantitis is mostly identified by level of the alveolar bone on the radiograph associated with clinical inflammation symptoms such as at least $>4 \mathrm{~mm}$ probing depth. ${ }^{8}$ The level of alveolar bone is considered a valuable parameter to signify the sternness of the peri-implantitis; nevertheless, it is difficult to know whether the changes of the monitored bone level are increasingly triggered by inflammation without the radiographic images and sequential observations. ${ }^{9}$

Several studies have revealed that peri-implantitis has various risk factors. ${ }^{10-12}$ Numerous periodontal-related factors are considered local; this prompts the bacterial existence at the implant site. It was concluded that fungal organisms were often recognized at healthy implant locations and peri-implantitis. ${ }^{13}$ Oral hygiene status ${ }^{14}$ and deep pockets ${ }^{15,16}$ are considered as some local risk indicators. Chronic periodontal disease ${ }^{17}$ and smoking ${ }^{10}$ are considered as general risk indicators that required frequent maintenance appointments. ${ }^{18-20}$

Many studies have been conducted to identify risk factors of peri-implantitis, but because of the indefinite diagnostic criteria of the condition, some ambiguity remains concerning its true prevalence and the nature of its risk factors. ${ }^{21}$ Some of the
${ }^{1-3,5}$ School of Dental Medicine, University of Nevada, Las Vegas, Nevada, USA

${ }^{4}$ School of Dental Medicine, Texas Tech University Health Sciences Center, Texas, USA

Corresponding Author: Neamat Hassan Abubakr, School of Dental Medicine, University of Nevada, Las Vegas, Nevada, USA, Phone: +1702-774-2307, e-mail: neamat.hassan@unlv.edu

How to cite this article: Anderson N, Lords A, Laux R, et al. Retrospective Analysis of the Risk Factors of Peri-implantitis. J Contemp Dent Pract 2020;21(12):1350-1353.

Source of support: Nil

Conflict of interest: None

most commonly indicated factors are periodontitis, tobacco use, cardiovascular disease, and other systemic diseases. Identifying and understanding these risk factors has the potential to allow earlier intervention and prevention of complications, thereby lowering the occurrence of peri-implantitis. The aim of the current study was to contribute to the literature in hopes of providing further clarification concerning risk factors of peri-implantitis.

\section{Materials and Methods Patient Sample and Data Collection}

This retrospective study was approved by University of Nevada, Las Vegas [UNLV] Biomedical Institutional Review Board and was given the status of exempt (IRB no. 1316816-1). Our data collection methods were inspired by a previous study where the data were collected from patients records in axiUm by using their clinical notes. ${ }^{22}$ A search for keyword in the clinical notes of patient charts in axiUm was conducted. The search terms were "peri-implantitis," 
"peri implantitis," and "perimplantitis" to identify the cases of documented peri-implantitis. All patient identifiers were removed from the collected data. The inclusion criteria for the patient population encompassed patients who were 18 years and older and were seen at UNLV, School of Dental Medicine clinics from January 2014 through September 2018 (Flowchart 1).

\section{Statistical Analysis}

The demographics of the data collected were analyzed using the Chi-square test.

\section{Results}

The reported patients by the clinician as peri-implantitis cases were 28 , with an overall of 45 implants. The distribution of males to females with peri-implantitis was 60.7 and $39.3 \%$, respectively. The highest number of patients (21.4\%) presenting with periimplantitis fell within age range of 65-69 years, followed by both aged groups 75-79 (14.3\%) and 80-84 (14.3\%) (Fig. 1). Total 53.3\% of the peri-implantitis were in the maxillary arch ( 24 implants), while $47.7 \%$ were in the mandibular region (21 implants) (Fig. 2). Results showed that the left mandibular first molar implant (15.6\%) was the predilection area for peri-implantitis followed by right mandibular first molar (8.9\%).

Flowchart 1: Descriptive data collection flowchart

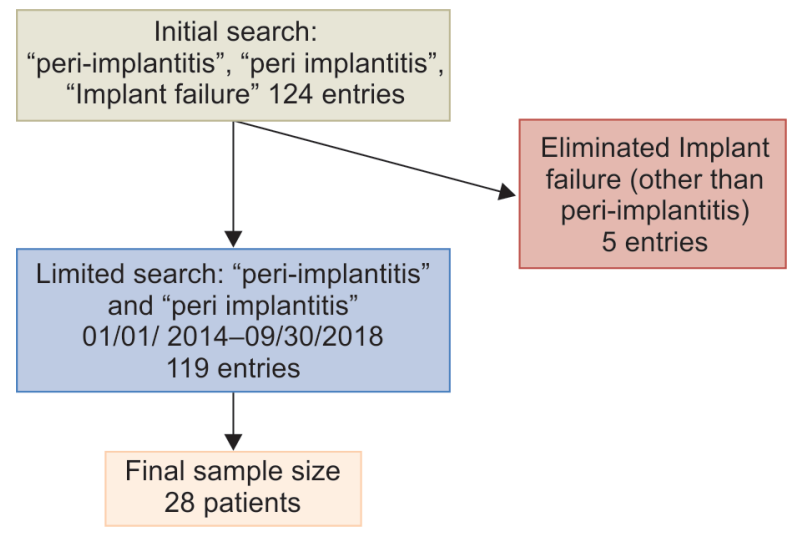

According to self-reported dental history, periodontitis (60.7\%) was the highest reported disease, while the most prevalent medical conditions in the studied population were respiratory diseases (42.9\%) followed by hypertension (28.6\%) and cancer (different types of cancer) (21.4\%) (Fig. 3). Results showed that peri-implantitis occurred most frequently among Caucasians (62.7\%), followed by Hispanics (29\%) and African Americans (11\%). Figure 4 shows mandibular cases of peri-implantitis.

\section{Discussion}

It has been typically accepted that periodontitis is a principal predictor of future peri-implantitis. ${ }^{23}$ Various clinical research reported and compared the incidence of peri-implantitis among periodontitis patients. ${ }^{24,25}$

The present findings were consistent with prior recent research, which indicated that chronic periodontitis is considered as a basic risk factor for peri-implantitis. ${ }^{26,27} \mathrm{~A}$ recent research identified that patients presented with severe periodontal disease had a substantial risk of peri-implantitis. ${ }^{26,27}$

There is limited reported research on the association between peri-implantitis and common systemic diseases. In 2014, Renvert et al. found a significant level of comorbidity of cardiovascular disease

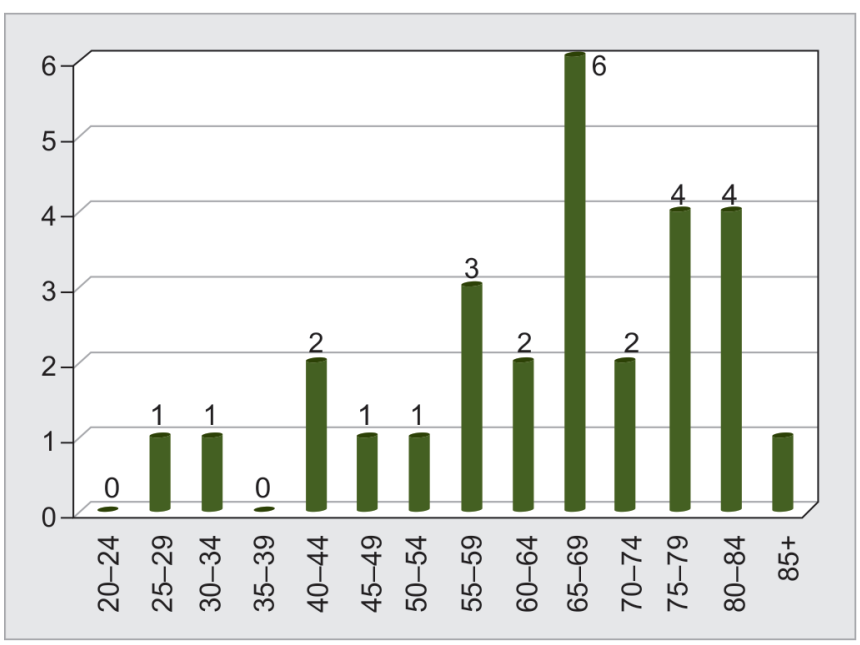

Fig. 1: Distribution of patients by age groups

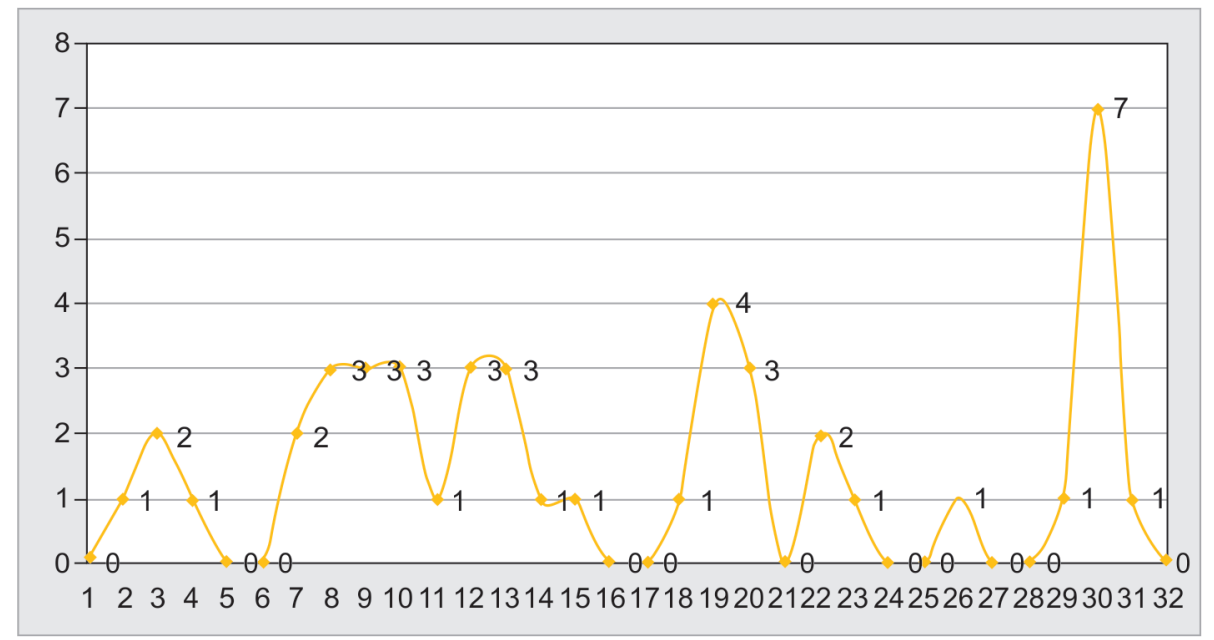

Fig. 2: Peri-implantitis cases distribution according to oral location 


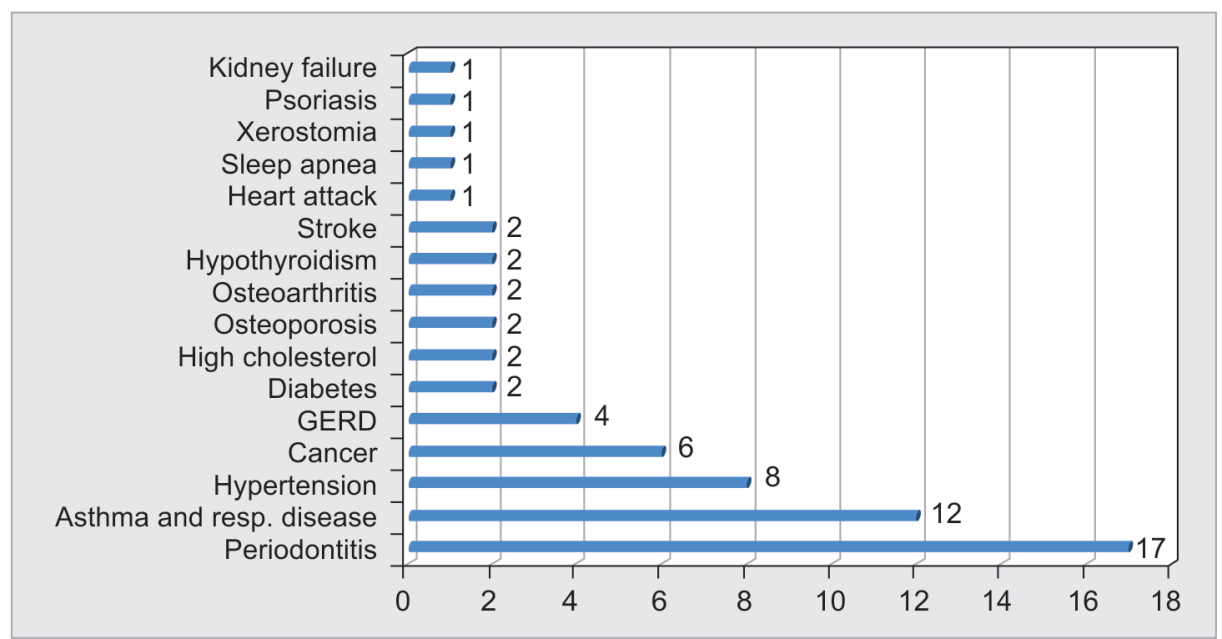

Fig. 3: The various diseases of the peri-implantitis patients
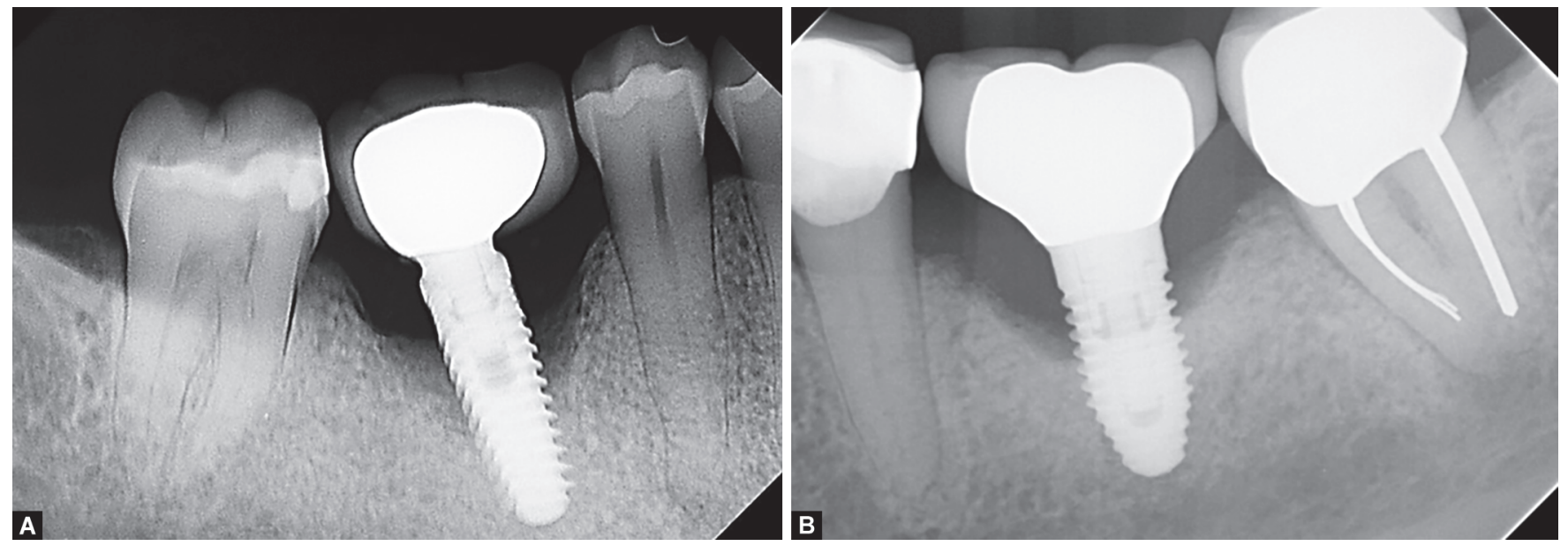

Figs 4A and B: Example of some of peri-implantitis cases

and periodontitis in the peri-implantitis cases. ${ }^{7}$ In the present study, an association between peri-implantitis and respiratory diseases (mainly included asthma) was the most commonly reported condition, followed by hypertension and cancer. Patients with uncontrolled diabetes experience compromised osseointegration; this will lead to a higher risk of peri-implantitis and implant failure. The disease duration impact is not well defined. ${ }^{28}$ In the present investigation, only $7 \%$ of the patients were reported as diabetic patients. The durability of peri-implantitis around the dental implant was linked to be a possible risk indicator for carcinogenesis. ${ }^{29}$ A recent cohort study indicated that survival analyzes showed an increased pattern of implant failure in cancer patients having radiotherapy and chemotherapy and being male but were not statistically significant. ${ }^{30}$ The present data indicated that $21.4 \%$ of the peri-implantitis cases were cancer patients and $50 \%$ of them were males.

The location of the implant has also been shown to have a correlation to peri-implantitis and implant failure. ${ }^{3}$ The present study revealed that peri-implantitis was found more frequently in the maxilla, although the difference was not statistically significant, which come into agreement with a previous research finding that indicated that jaw of treatment is a predictor for peri-implant bone loss. ${ }^{31-33}$
Other factors such as race also aligned with prior studies with peri-implantitis occurring most frequently in Caucasians, followed by Hispanics and African Americans-in spite the major clinical population was considered to be Hispanics population. One major limitation to be considered is that the data were self-reported by the patients-and spanned for only 4 years. An extended follow-up period would be constructive in gathering a more complete representation of the occurrence of peri-implantitis case, which is reported by patients and clinicians.

\section{Conclusion}

Within the limitations of the present investigation, findings support previous claims that periodontitis remains the strongest predictor of peri-implantitis. Also, it was concluded that a possible correlation might exist between peri-implantitis and the location of the implant, patient age, and respiratory diseases.

\section{References}

1. Dodson TB. Predictors of dental implant survival. J Mass Dent Soc 2006;54:34-38.

2. Anitua $E$, Orive $G$, Aguirre JJ, et al. 5-year clinical experience with BTI dental implants: risk factors for implant failure. J Clin Peri 2008;35(8):724-732. DOI: 10.1111/j.1600-051X.2008.01248.x. 
3. Jemt $\mathrm{T}$, Olsson $\mathrm{M}$, Renouard $\mathrm{F}$, et al. Early implant failures related to individual surgeons: an analysis covering 11,074 operations performed during 28 years. Clin Implant Dent and Rel Res 2016;18(5):861-872. DOI: 10.1111/cid.12379.

4. Rakic M, Galindo-Moreno P, Monje A, et al. How frequent does periimplantitis occur? A systematic review and meta-analysis. Clin Oral invest 2018;22(4):1805-1816. DOI: 10.1007/s00784-017-2276-y.

5. Lindhe J, Meyle J. Peri-implant disease: consensus report of the sixth European workshop on periodontology. J Clin Peri 2008;35:282-285. DOI: 10.1111/j.1600-051X.2008.01283.x.

6. Mombelli A, Müller N, Cionca N. The epidemiology of periimplantitis. Clin Oral Implants Res 2012;23:67-76. DOI: 10.1111/j.16000501.2012.02541.x.

7. Renvert $\mathrm{S}$, Aghazadeh $\mathrm{A}$, Hallstrom $\mathrm{H}$, et al. Factors related to peri-implantitis-a retrospective study. Clin Oral Implants Res 2014;25(4):522-529. DOI: 10.1111/clr.12208.

8. Sanz I, Garcia-Gargallo M, Herrera D, et al. Surgical protocols for early implant placement in post-extraction sockets: a systematic review. Clin Oral Implants Res 2012;23:67-79. DOI: 10.1111/j.16000501.2011.02339.x.

9. Lee $C T$, Huang YW, Zhu L, et al. Prevalence of peri-implantitis and peri-implant mucositis: systematic review and meta-analysis. J Dent 2017;62:1-12. DOI: 10.1016/j.jdent.2017.04.011.

10. Heitz-Mayfield LJ. Peri-implant diseases: Diagnosis and risk indicators. J Clin Perio 2008;35:292-304. DOI: 10.1111/j.1600-051X.2008.01275.x.

11. Jepsen $S$, Berglundh $T$, Genco R, et al. Primary prevention of peri-implantitis: managing peri-implant mucositis. J Clin Perio 2015;42:S152-S157. DOI: 10.1111/jcpe.12369.

12. Renvert S, Quirynen M. Risk indicators for peri-implantitis. A narrative review. Clin Oral Implants Res 2015;26:15-44. DOI: 10.1111/clr.12636.

13. Schwarz F, Becker $K$, Rahn S, et al. Real-time PCR analysis of fungal organisms and bacterial species at peri-implantitis sites. Int J Implant Dent 2015;1(1):9. DOI: 10.1186/s40729-015-0010-6.

14. Serino G, Ström C. Peri-implantitis in partially edentulous patients: association with inadequate plaque control. Clin Oral Implants Res 2009;20(2):169-174. DOI: 10.1111/j.1600-0501.2008.01627.x.

15. Mombelli A, Marxer M, Gaberthüel T, et al. The microbiota of osseointegrated implants in patients with a history of periodontal disease. J Clin Perio 1995;22(2):124-130. DOI: 10.1111/j.1600-051X.1995. tb00123.x.

16. Seki K, Nakabayashi S, Tanabe N, et al. Correlations between clinical parameters in implant maintenance patients: Analysis among healthy and history-of-periodontitis groups. Int J Implant Dent 2017;3(1):45. DOI: 10.1186/s40729-017-0108-0.

17. Renvert S, Persson GR. Periodontitis as a potential risk factor for peri-implantitis. J Clin Perio 2009;36:9-14. DOI: 10.1111/j.1600051X.2009.01416.x.

18. Costa FO, Takenaka-Martinez S, Cota LM, et al. Peri-implant disease in subjects with and without preventive maintenance: a 5 -year follow-up. J Clin Perio 2012;39(2):173-181. DOI: 10.1111/j.1600051X.2011.01819.x.

19. Monje A, Aranda L, Diaz KT, et al. Impact of maintenance therapy for the prevention of peri-implant diseases: a systematic review and meta-analysis. J Dent Res 2016;95(4):372-379. DOI: 10.1177/0022034515622432.

20. Pjetursson $B E$, Thoma $D$, Jung $R$, et al. A systematic review of the survival and complication rates of implant-supported fixed dental prostheses (FDPs) after a mean observation period of at least 5 years. Clin Oral Implants Res 2012;23:22-38. DOI: 10.1111/j.16000501.2012.02546.x.

21. Atieh $M$, Alsabeeha $N$, Faggion $C$, et al. The frequency of periimplant diseases: a systematic review and meta-analysis. J of Perio 2012;84:1586-1598. DOI: 10.1902/jop.2012.120592.

22. Sturgill R. Prevalence and clinical characteristics of teeth extracted with a diagnosis of cracked tooth: a retrospective study. VCU Scholars Compass 2017. 1-450.

23. Levin L, Ofec R, Grossmann $Y$, et al. Periodontal disease as a risk for dental implant failure over time: a long-term historical cohort study. J Clin Perio 2011;38(8):732-737. DOI: 10.1111/j.1600-051X.2011.01745.x.

24. Karoussis IK, Salvi GE, Heitz-Mayfield LJ, et al. Long-term implant prognosis in patients with and without a history of chronic periodontitis: a 10-year prospective cohort study of the ITI dental implant system. Clin Oral Implants Res 2003;14(3):329-339. DOI: 10.1034/j.1600-0501.000.00934.x.

25. Schou S, Holmstrup P, Worthington HV, et al. Outcome of implant therapy in patients with previous tooth loss due to periodontitis. Clin Oral Implants Res 2006;17(S2):104-123. DOI: 10.1111/j.16000501.2006.01347.x.

26. Arunyanak SP, Sophon N, Tangsathian T, et al. The effect of factors related to periodontal status toward peri-implantitis. Clin Oral Implants Res 2019;30(8):791-799. DOI: 10.1111/clr.13461.

27. Marcantonio C, Nicoli LG, Marcantonio Junior E, et al. Prevalence and possible risk factors of peri-implantitis: a concept review. J Contemp Dent Pract 2015;16(9):750-757. DOI: 10.5005/jp-journals10024-1752.

28. Naujokat $\mathrm{H}$, Kunzendorf $\mathrm{B}$, Wiltfang J. Dental implants and diabetes mellitus—a systematic review. Inter J Implant Dent 2016;2(1):5. DOI: 10.1186/s40729-016-0038-2.

29. Noguchi $M$, Tsuno $H$, Ishizaka $R$, et al. Primary peri-implant oral intraepithelial neoplasia/carcinoma in situ: a case report considering risk factors for carcinogenesis. Inter J Implant Dent 2017;3(1):47. DOI: 10.1186/s40729-017-0109-z.

30. Laverty DP, Addison O, Wubie BA, et al. Outcomes of implantbased oral rehabilitation in head and neck oncology patients-a retrospective evaluation of a large, single regional service cohort. Inter J Implant Dent 2019;5(1):8. DOI: 10.1186/s40729-019-0161-y.

31. Vervaeke S, Collaert B, Cosyn J, et al. A multifactorial analysis to identify predictors of implant failure and peri-implant bone loss. Clin Implant Dent Relat Res 2015;17:e298-e307. DOI: 10.1111/cid.12149.

32. Mengel R, Heim T, Thöne-Mühling M. Mucositis, peri-implantitis, and survival and success rates of oxide-coated implants in patients treated for periodontitis 3-to 6-year results of a case-series study. Int J Impl Dentis 2017;3(1):48. DOI: 10.1186/s40729-017-0110-6.

33. Mameno $T$, Wada M, Onodera $Y$, et al. Longitudinal study on risk indicators for peri-implantitis using survival-time analysis. J Prosthodon Res 2019;63(2):216-220. DOI: 10.1016/j.jpor.2018.12.002. 